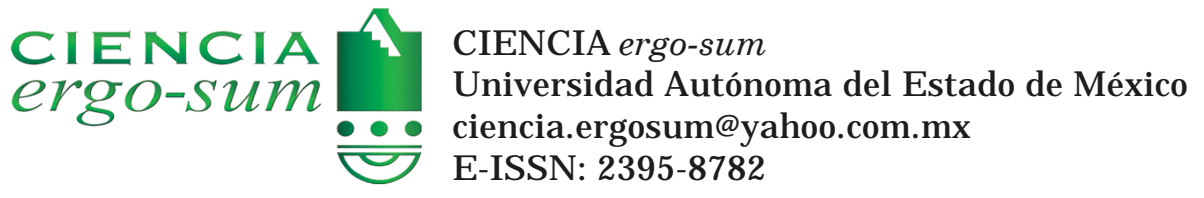

\title{
Rol del interventor en la gestión de proyectos de infraestructura pública: estudio de caso del Valle del Cauca, Colombia
}

Chamorro Solórzano, Gerardo Esteban; Pérez Ruíz, Diego Darío; Serrano Guzmán, María Fernanda Rol del interventor en la gestión de proyectos de infraestructura pública: estudio de caso del Valle del Cauca, Colombia CIENCIA ergo-sum, vol. 29, núm. 1, marzo-junio 2022 | e145 Ciencias Sociales

Universidad Autónoma del Estado de México, México Esta obra está bajo una Licencia Creative Commons Atribución-NoComercial-SinDerivar 4.0 Internacional.

$$
\text { (c) }(\$
$$

Chamorro Solórzano, G. E., Pérez Ruíz, D. D. y Serrano Guzmán, M. F. (2022). Rol del interventor en la gestión de proyectos de infraestructura pública: estudio de caso del Valle del Cauca, Colombia. CIE NCIA ergo-sum, 29(1). https://doi .org/10.30878/ces.v29n1a1 


\title{
Rol del interventor en la gestión de proyectos de infraestructura pública: estudio de caso del Valle del Cauca, Colombia
}

\section{The role of the auditor in the management of public infrastructure projects: a case study of Valle del Cauca, Colombia}

\author{
Gerardo Esteban Chamorro Solórzano \\ Federación Nacional de Cafeteros de Colombia, Colombia \\ gerardoesteban.chamorro@gmail.com \\ (D) http://orcid.org/0000-0002-3820-0660 \\ Diego Dario Pérez Ruiz \\ Pontificia Universidad Javeriana Cali, Colombia \\ ddperez@javerianacali.edu.co \\ (D) http://orcid.org/0000-0002-9656-2803 \\ María Fernanda Serrano Guzmán \\ Pontificia Universidad Javeriana Cali, Colombia \\ maria.serrano@javerianacali.edu.co \\ (1) https://orcid.org/0000-0002-7366-6597
}

\section{RESUMEN}

Se analiza el papel del interventor en los contratos de interventoría adelantados en el Valle del Cauca en un periodo posterior a la expedición del Estatuto Anticorrupción. Se revisó un total de 64 contratos del Concurso de Méritos. El estudio reveló que hay variedad en los alcances de desempeño del interventor en contratos de objetos contractuales similares y se señalan los vacíos en la legislación actual y las inconsistencias en requisitos habilitantes. Se incluyen también recomendaciones generales sobre procedimientos adecuados para la contratación de interventoría. El rol del interventor en Colombia como veedor de los términos pactados en los contratos de infraestructura pública es fundamental para el cumplimiento del objeto contractual.

Palabras Claves: contratación estatal, interventor, rol.

\section{Abstract}

We analyze the role of the auditor in the contracts done in Valle del Cauca, in a period after the issuance of the Anti-Corruption Statute. A total of 64 Merit Contest contracts signed were reviewed. The study revealed that there is a variety in the scope of performance of the financial auditor in contracts with similar contractual objects, the gaps in the current legislation and inconsistencies in financial requirements. General recommendations are also included on appropriate procedures for contracting auditing. The role of the auditor in Colombia as overseer of the terms agreed in public infrastructure contracts is essential for the fulfillment of the contractual object.

KEYWORDS: State contracting, auditor, role.

\section{INTRODUCCIÓN}

La administración pública responde a las necesidades de infraestructura de las comunidades mediante la ejecución de proyectos que suplen obras, bienes o servicios (Serrano Guzmán, Carmona Escobar, Pérez 
Ruíz et al., 2019), los cuales pueden requerir o no del concurso de un interventor. En Colombia, país en vía de desarrollo, una persona natural o jurídica es la encargada de la labor de interventoría, que consiste en vigilar el desarrollo de los proyectos (Universidad Industrial de Santander, 2014), públicos o privados, contratados bien sea durante la etapa de estudios y diseños y de la construcción o durante los estudios, diseños y construcción de los proyectos (Servicio Electrónico de Contratación Pública, 2016). Justamente, la ausencia o el desconocimiento de las recomendaciones de la interventoría han repercutido en hechos nefastos en territorio colombiano como el colapso del edifico Space en Medellín (Alcaldía de Medellín, 2014) o el retraso en la terminación de las obras de la troncal de Transmilenio (Semana, 2010) en Santa Fe de Bogotá, por citar algunos ejemplos. Asimismo, se presume que probablemente no se hubiesen dado los hallazgos administrativos y disciplinarios encontrados en el Informe de Actuación Especial No. 018 de la ejecución de recursos del Fondo Nacional de Regalías y el Sistema General de Regalías (Contraloría General de La República, 2014).

En general, puede afirmarse que la falta de injerencia de la interventoría ha desencadenado en el incumplimiento de cronogramas y falta de control en la ejecución de las obras (National Research Council Canada, 2009), hechos que terminan por generar sobrecostos por errores y ponen en evidencia omisiones de los consultores (Ottawa Documents, 2012). Precisamente, la falta de posturas claras sobre la gestión del interventor ha derivado en la existencia de manuales particulares para el ejercicio de esta labor en entidades gubernamentales en Colombia con las implicaciones de libre interpretación que esto puede acarrear tanto para el ente contratante como para el ejecutor del contrato.

Con base en este contexto, se hace una revisión de casos de contratos de infraestructura pública en la modalidad de Concurso de Méritos, los cuales están relacionados con la contratación de labores de interventoría en estado liquidado al 31 de diciembre de 2016 y ejecutados en el Valle del Cauca (Colombia) en un periodo posterior a la expedición del estatuto anticorrupción, además de identificar responsabilidades que abarcan aspectos de planeación (Chamorro Solórzano, 2019). Para ello se realizó una consulta en el sistema electrónico de contratación pública (SECOP I), instrumento digital que emplean las entidades estatales en Colombia para la postulación de ofertas contractuales de productos, bienes y servicios en esta nación al cual pueden acceder los ciudadanos para la consulta y postulación de propuestas para contratación o simplemente para veeduría de los procesos que tienen información allí almacenada. Aunado a lo anterior, se revisaron las leyes y decretos que rigen esta actividad; este análisis permitió la identificación de similitudes y vacíos en los alcances en las diferentes actividades que lleva a cabo el interventor. Al final, se proponen unas recomendaciones generales sobre procedimientos adecuados para la contratación durante el ciclo de vida de un proyecto de interventoría buscando que, con la divulgación de estos resultados, los expertos en aspectos legislativos tengan argumentos para la postulación de una normativa más precisa.

\section{Metodología}

La investigación para este artículo es de carácter descriptivo, cualitativo y cuantitativo. Para el levantamiento de los hallazgos del estudio se siguieron las siguientes etapas. Se revisó la normatividad vigente en Colombia para el ejercicio de la contratación estatal y, en lo particular, de las labores de la interventoría. Se llevó a cabo la búsqueda de los contratos de interventoría bajo la modalidad de contratación Concurso de Méritos durante la construcción de proyectos en estado liquidado en el SECOP como se hizo en Serrano Guzmán, Carmona Escobar, Pérez Ruíz et al. (2019). El periodo se consideró desde la expedición del estatuto anticorrupción (Congreso de Colombia, 2011) hasta el 31 de diciembre de 2016. Esta clasificación arrojó 64 contratos. Una vez realizado el filtro de estos procesos, se procede a revisar la documentación existente. Finalmente, se presentan recomendaciones para acciones futuras en la gestión de proyectos de obras públicas en los cuales se requiere el concurso del interventor. 


\section{Normatividad LegaL}

A partir del 15 de enero de 2018 surge la Ley 1882 que establece disposiciones para fortalecer la contratación pública en Colombia (Congreso de Colombia, 2018). Sin embargo, a la fecha en que se lleva a cabo esta investigación, la contratación se encontraba amparada principalmente por la Ley 80 de 1993, la cual expide el Estatuto General de Contratación de la Administración Pública (Congreso de Colombia, 1993), cuyo artículo 32, inciso segundo, menciona que la interventoría es un contrato de consultoría, y por la Ley 1474 de 2011 (Congreso de Colombia, 2011)[1] en donde se establecen los mecanismos de prevención, investigación y sanción de actos de corrupción y la efectividad del control de la gestión pública.

En el ámbito colombiano, la falta de normatividad particular para el ejercicio de la interventoría ha motivado que las entidades públicas establezcan sus propios manuales de contratación y, por ende, aquella relacionada a la contratación específica de los servicios de interventoría (figura 1).

En esencia, las leyes establecen la necesidad de la vigilancia y control en aspectos técnicos, administrativos, contables, legales, económicos, ambientales, de seguridad industrial, salud ocupacional y control de calidad de los proyectos (Sánchez Henao, 2007), labor que puede ser ejecutada mediante la interventoría, aunque sobra decir que la presencia de ésta no garantiza la calidad de la obra (Gorbaneff, González y Barón, 2011).[2] Por tal razón, históricamente en Colombia se han expedido varios decretos y leyes que intentan regir la contratación de servicios y bienes con el Estado (figura 2).

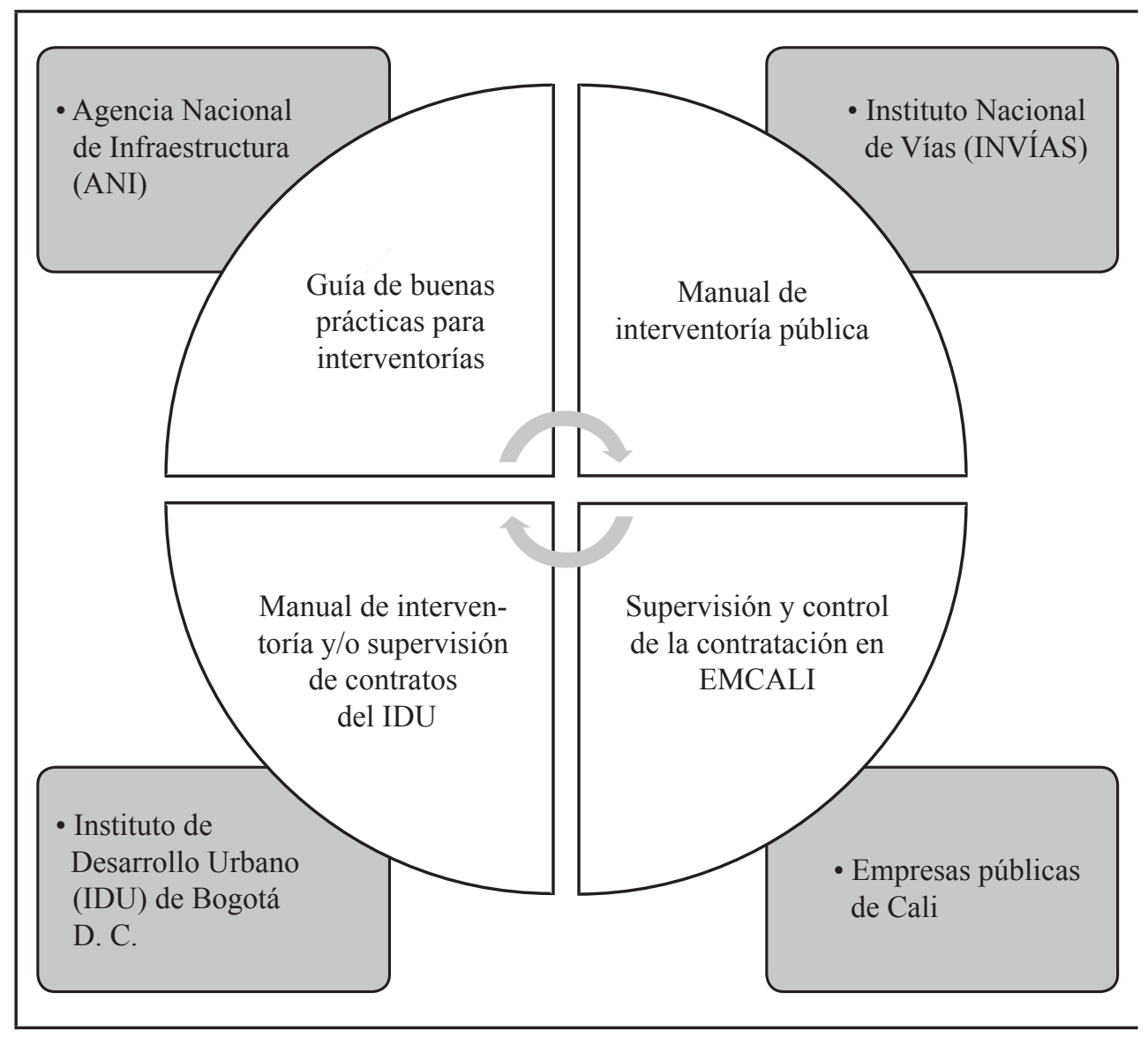

FIGURA 1

Manuales de interventoría en diferentes instituciones

Fuente: Agencia Nacional de Infraestructura (2013), Instituto Nacional de Vías (2010), Empresas Municipales de Cali (2014), Instituto de Desarrollo Urbano (2015). 


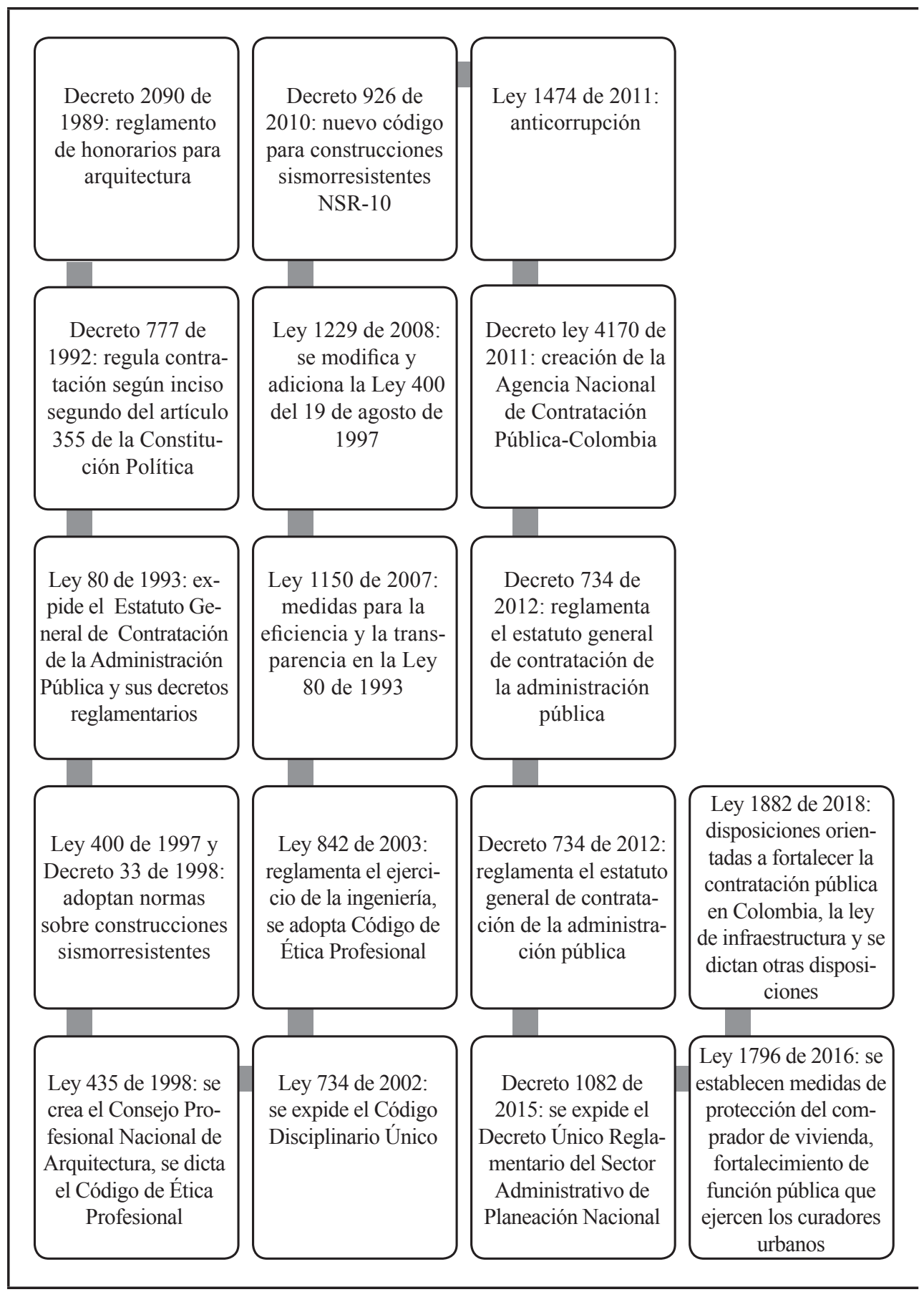

FIGURA 2

Leyes y decretos establecidos en Colombia para la contratación pública

\section{Resultados}

Se presenta una revisión sucinta de la normatividad legal existente que Chamorro Solórzano (2019) identificó en las leyes y decretos que en Colombia rigen la labor del interventor. En este apartado se hacen señalamientos que revelan los vacíos existentes. 


\section{1. Decreto 2090 de 1989}

Incluye la definición de interventoría, costos de honorarios y establece su responsabilidad. Dentro de los vacíos encontrados en este decreto está el tipo de profesionales que deben intervenir en el esquema básico, anteproyecto y construcción, la definición puntual de la labor de la interventoría y de los perfiles de desempeño de quienes la realizan, así como la injerencia en las decisiones del proyecto.

\section{2. Decreto 777 de 1992}

Determina un monto máximo del $5 \%$ para honorarios por servicios de interventoría ejecutados en convenios de cooperación, cofinanciación o asociación con empresas privadas sin ánimo de lucro. Esto conlleva a que no se consideren los costos reales de la actividad.

\section{3. Ley 80 de 1993}

No se establecen lineamientos específicos para la labor de interventoría. Dado que este ejercicio forma parte de la Administración Pública, debería estar contemplado este análisis en esa ley.

\subsection{Ley 400 de 1997}

Define a los profesionales que pueden desempeñarse como interventores y se plantean aspectos de este ejercicio. Aunque no se menciona el tipo de obras que requieren supervisión técnica continua, sí se establece el requerimiento de estos servicios profesionales en función del área que se va a construir para aquellas obras que tengan más de $2000 \mathrm{~m}^{2}$ de área. Asimismo, contempla experiencia general mayor a cinco años y específica en cuanto a que el interventor debió haberse desempeñado en diferentes ramas de la construcción para ejercer como supervisor técnico.

\section{5. Ley 435 de 1998}

Se menciona la ética profesional frente a las situaciones señaladas en ella, lo cual limita la universalidad de su aplicación. Los procedimientos disciplinarios establecen la cancelación de la matrícula profesional que aplica a los arquitectos y profesiones auxiliares.

\subsection{Ley 734 de 2002}

Aunque no está dirigida a los interventores, sí menciona las causales de faltas disciplinarias en las que puede incurrir quien realice actividades de interventoría.

\section{7. Ley 842 de 2003}

Esta ley es similar a la Ley 435 de 1998. Sin embargo, deja vacíos en el tipo de profesionales que pueden ejercer como interventores de proyectos de construcción, por lo que queda abierto el ejercicio de interventoría a cualquier tipo de disciplina. Además, esta ley establece las sanciones por faltas al ejercicio de las funciones y profundiza en el comportamiento como profesional ante diferentes situaciones que se puedan presentar. 


\section{8. Ley 1150 de 2007}

Establece de manera taxativa las modalidades de contratación en el caso de procesos con entidades públicas, pero no hace señalamientos puntuales para labores de interventoría o supervisión.

\section{9. Ley 1229 de 2008}

Señala las profesiones habilitadas para el ejercicio de labores de interventoría, pero no es taxativa en alcances y responsabilidades y no establece diferencias entre los conceptos de interventor y supervisor técnico.

\section{10. Ley 1474 de 2011}

Es el estatuto anticorrupción en donde se encuentran las inhabilidades y las sanciones por faltas relacionadas con corrupción en el sector privado y público. Se plantean de manera explícita los roles del supervisor y del interventor, además dicta que el interventor aparecerá únicamente en el momento en que se requiera una figura especializada en el ámbito técnico y el supervisor sí debe estar en todas partes del proyecto. Como se observa, existe diferenciación tanto para el rol de interventor como del supervisor.

\section{11. Decreto 734 de 2012}

En este decreto se enfatiza la importancia de conocer los riesgos asociados a la contratación y las modalidades existentes para contratación de servicios de interventoría.

\section{12. Decreto 1510 de 2013}

Se enfatiza en la relevancia de incluir a la interventoría en los pliegos de condiciones.

\section{13. Decreto 1082 de 2015}

Se hace hincapié en la relevancia de mantener publicaciones en el SECOP de los procesos de contratación. Se mencionan también algunas de las responsabilidades de la interventoría, principalmente en aspectos de tipo administrativo y financiero.

\section{14. Ley 1796 de 2016}

Contempla la necesidad de supervisión técnica dependiendo del área de la construcción. Hace una modificación a la Ley 1474 de 2011 y asigna responsabilidad civil, fiscal, penal y disciplinaria a quienes ejerzan contratos de interventoría. Se ratifican los grandes retos que tiene quien ejerce la interventoría. Adicionalmente, se estipulan documentos tipo para la contratación de las interventorías.

Como puede observarse, son múltiples las disposiciones y los intentos por legislar y normalizar el ejercicio del interventor. Y, aun así, es común encontrar situaciones de modificaciones de cronograma o de presupuesto o de ambos. En un total de 10 procesos de los 64 analizados se cumplieron los tiempos de ejecución y la labor de interventoría se ejecutó al precio pactado (cuadro 1). El 80\% de los procesos contemplaban alcance técnico, administrativo, financiero y tan sólo el $50 \%$ contemplaba alcance jurídico o legal. Estos procesos fueron cerrados de manera normal. 


\section{CUADRO 1}

Procesos sin modificaciones en tiempo ni presupuesto*

\begin{tabular}{|c|c|c|c|c|c|c|}
\hline \multirow{2}{*}{ Número del proceso } & \multicolumn{5}{|c|}{ Alcance de la interventoría } & \multirow{2}{*}{ Fuente } \\
\hline & Tec. & Adm. & Fin. & Con. J. y/o lg. Amb. & Soc. Int. Gen. & \\
\hline $330-15-08-027$ & $\mathrm{X}$ & $\mathrm{X}$ & $\mathrm{X}$ & $\mathrm{X}$ & & SECOP I, 2015a \\
\hline 4133.0.32.076-2014 & $\mathrm{X}$ & $\mathrm{X}$ & $X$ & $\mathrm{X}$ & & SECOP I, 2014a \\
\hline MC-CM-007-2011 & $\mathrm{X}$ & $\mathrm{X}$ & $\mathrm{X}$ & & & SECOP I, 2011a \\
\hline $330-015-008-022$ & $\mathrm{X}$ & $\mathrm{X}$ & $\mathrm{X}$ & $\mathrm{X}$ & & SECOP I, 2013a \\
\hline MV-CM-002 DE 2013 & $\mathrm{X}$ & $\mathrm{X}$ & $\mathrm{X}$ & $\mathrm{X}$ & $\mathrm{X}$ & SECOP I, 2013b \\
\hline CM-FEV-001-2013 & $\mathrm{X}$ & $\mathrm{X}$ & $X$ & $\mathrm{X}$ & & SECOP I, 2013c \\
\hline FEV-CM-003-2015 & $\mathrm{X}$ & $\mathrm{X}$ & $\mathrm{X}$ & $\mathrm{X}$ & & SECOP I, 2015b \\
\hline $\begin{array}{l}\text { 001-2013-OOPP-CONCURSO } \\
\text { DE MERITOS }\end{array}$ & $\mathrm{X}$ & & & & & SECOP I, 2013d \\
\hline $2013-187$ & & & & & $\mathrm{X}$ & SECOP I, 2013e \\
\hline $2015-154$ & $\mathrm{X}$ & $\mathrm{X}$ & $X$ & & & SECOP I, 2015c \\
\hline
\end{tabular}

Fuente: elaboración propia.

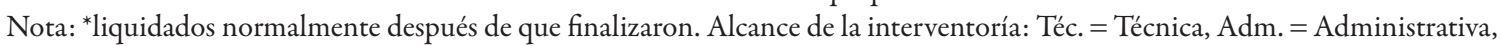
Fin. $=$ Financiera, Con. $=$ Contable, J. y/o lg. $=$ Jurídica y $/$ o legal, Amb. $=$ Ambiental, Soc. $=$ Social, Int. $=$ Integral, Gen. $=$ General .

Un total de tres procesos de los 64 analizados presentó adiciones presupuestales, pero no se dieron modificaciones en tiempo (cuadro 2).

\section{CUADRO 2}

Procesos sin modificaciones en tiempo y con adiciones presupuestales $(\%)^{*}$

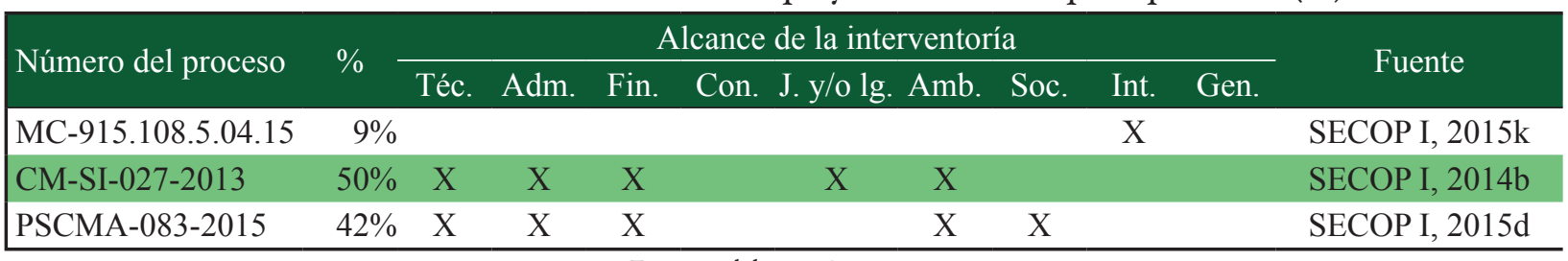

Fuente: elaboración propia.

Nota: ${ }^{*}$ liquidados normalmente después de que finalizaron.

Referente al cuadro 3 se puede apreciar que 18 de los 64 procesos liquidados presentaron adiciones presupuestales y modificaciones en el tiempo de ejecución y, en su mayoría, el alcance de la interventoría abarcó aspectos técnicos, administrativos y financieros ( $97.4 \%$ de los casos).

En el cuadro 4 se aprecian procesos de interventoría que se adelantaron en menor tiempo de lo pactado, en tres de los cuales no se requirió de adición presupuestal. En todos estos procesos se requería interventoría en aspectos técnicos, administrativos y financieros. Por las particularidades de los estudios contratados, solamente un proceso contrató interventoría ambiental y social. Por otro lado, este análisis demostró que el proceso 4133.0.32.020-2014 (SECOP I, 2014e) fue ejecutado en un menor tiempo (27\%) y con un ahorro del $4 \%$.

Tan sólo el proceso PSCMA-071-2015 (SECOP I, 2015h) se liquidó unilateralmente y tuvo un aumento de duración del 32\%. Este proceso tuvo un alcance técnico, administrativo, financiero, ambiental y social. Por su parte, los procesos SECOP I (2015b), SECOP I (2015k) y SECOP I (2013e) se ejecutaron con un alcance de interventoría integral, mientras que SECOP I (2014a, 2014e), SECOP I (2011) tuvieron un alcance general. Se presume que los conceptos integral y general deben ser interpretados bajo el entendido de que en estas denominaciones se están incorporando los alcances técnicos, administrativos, financieros, contables, jurídicos y/o legal, ambiental y social. Sin embargo, dejar esta interpretación a juicio del oferente es un riesgo al momento de protocolizar el contrato. 


\section{CUADRO 3}

Procesos con adición presupuestal (\%) y cambios de duración (\%)

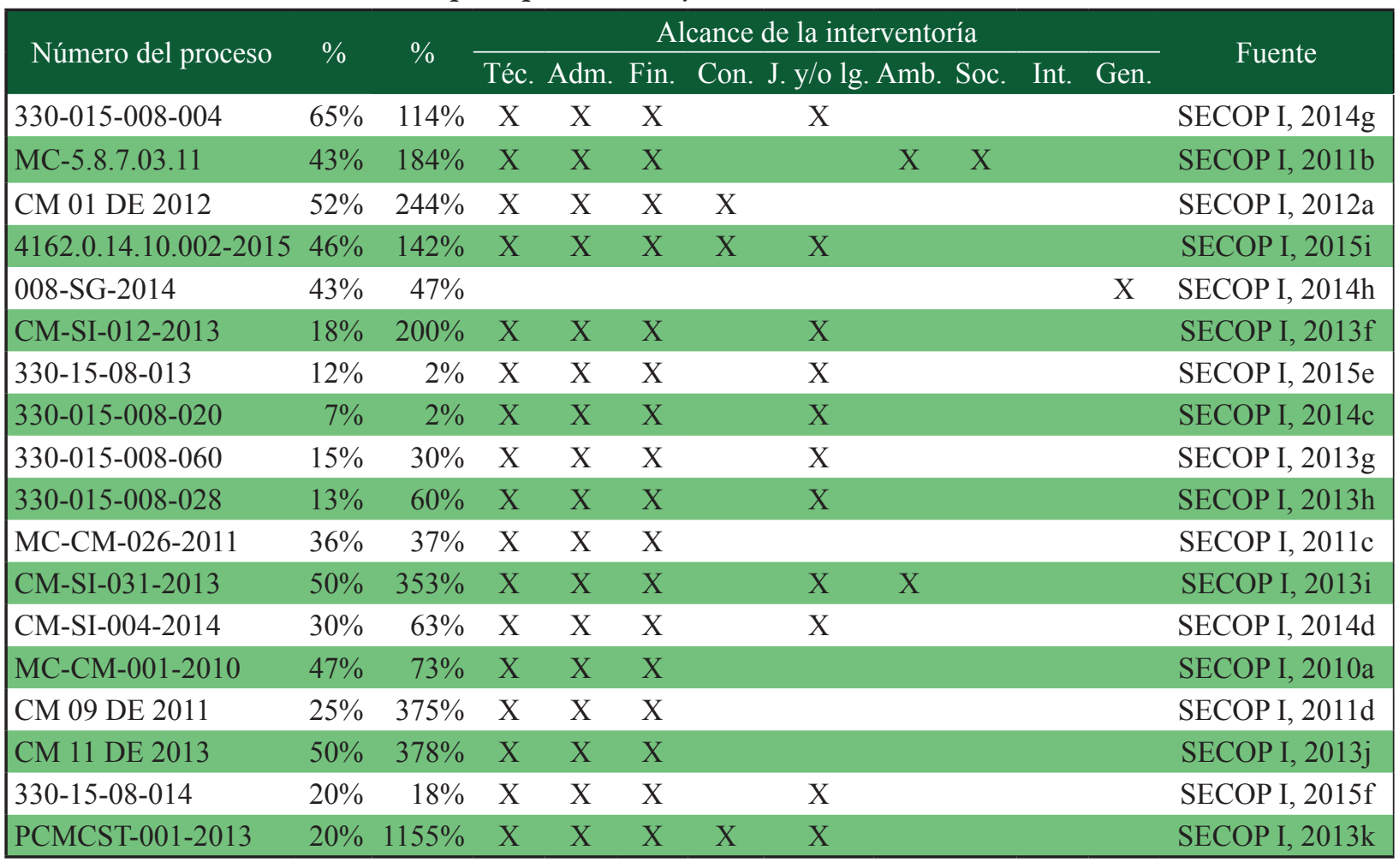

Fuente: elaboración propia.

Nota: alcance de la interventoría: Tec. $=$ Técnica, Adm. = Administrativa, Fin. = Financiera, Con. = Contable, J. y lg. = Jurídica y/o legal, Amb. = Ambiental, Soc. $=$ Social, Int. = Integral, Gen. $=$ General.

\section{CUADRO 4}

Procesos ejecutados en menor tiempo (\%) y en menor costo (\%)

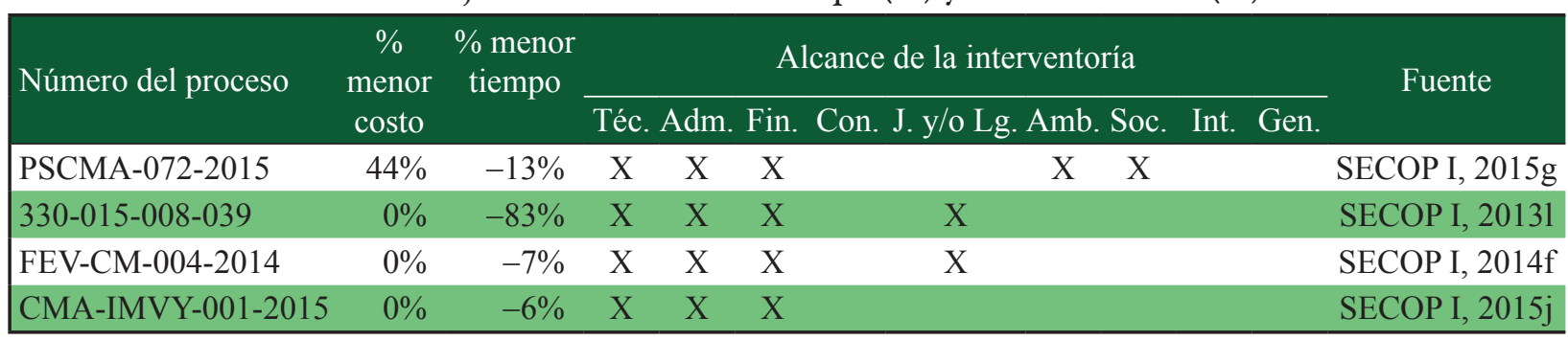

Fuente: elaboración propia.

En lo que corresponde a este estudio se encontró que en 57 de los 64 contratos analizados se contempló el requerimiento de interventoría técnica. Además, en 54 contratos se empleó la interventoría administrativa, la cual, se presume, debería incluir aspectos financieros, jurídicos y/o legales, ambientales, sociales, contables. También, se debe hacer mención que en cuatro contratos se menciona un alcance general y en otros tres el alcance fue integral, pero los conceptos asociados a ambos tipos de alcances son similares de modo que podría considerarse como un solo rol de interventor en donde, aunado a los aspectos administrativos descritos, deberían explícitamente incorporarse los aspectos técnicos. A pesar del detalle para la descripción de los alcances en los contratos analizados, se presentan variaciones significativas durante la ejecución y entrega de los proyectos. Este hecho corrobora que, sin lugar a dudas, durante el ciclo de vida de un proyecto es necesario un ejercicio de supervisión de modo que se dé cumplimiento a los compromiso contractuales en las diferentes etapas y 
propender con ello por el principio de transparencia (Serrano Guzmán, Carmona Escobar, Pérez Ruíz et al., 2019), además de defender las inversiones de los fondos del erario; sin embargo, la revisión de los procesos de este estudio demuestran que existen falencias desde la etapa de planeación por cuanto en repetidos casos se incurrió en adiciones presupuestales o adiciones de tiempo o ambos, contratos ejecutados a un menor costo, atrasos derivados de diseños incompletos, entre otros. En específico, algunos de los hallazgos que corroboran la falta de planeación son los siguientes:

- Mayor alcance en la ejecución del proyecto contratado en un inicio. En este caso, a escasos dos meses de firmada la adición del contrato por mayor cantidad de obra, se suscribió la Modificatoria 3 que enunciaba una nueva adición y prórroga (SECOP I, 2014g). En este caso es evidente que se desconocían los alcances reales de la obra que se estaba contratando.

- La ausencia o baja calidad de los estudios y diseños del proyecto que conllevaron al aumento de costos y tiempo de ejecución (SECOP I, 2014g). Situación similar en el proceso SECOP I (2012a) en donde los estudios y diseños estuvieron alejados de las normas técnicas y las especificaciones de construcción, además que se presentaron adiciones de obra y suspensión del contrato. [3]

- Uso de fondos de anticipo para destinos diferentes a los establecidos en la cláusula del contrato[4] (SECOP I, 2012a), situación presentada por falta de presencia del interventor y del supervisor del contrato (Chamorro Solórzano, 2019).

- Adición presupuestal para dar cumplimiento a condiciones de puesta en marcha de la obra entregada (SECOP I, 2015i). Se presume que la interventoría desconocía requisitos para dar cumplimiento a las especificaciones técnicas de la obra contratada.

- Desequilibrio económico con prejuicio para el contratista (SECOP I, 2014i). Situaciones como ésta pueden desencadenar una demanda para el ente contratante. [5]

- Dificultades para la entrega de la obra debido a debilidades en el ejercicio de la interventoría a quien se le solicitó en el Acta de Liquidación que debía “realizar seguimiento acompañamiento al contratista” (SECOP I, 2014h). Esta situación representa inconvenientes para la comunidad que no puede disfrutar del inmueble objeto del contrato.

Por otro lado, aunque en la Ley 1796 de 2016 se establece el cumplimiento de unos documentos tipo para quienes realicen labores de interventoría, en los términos de referencia de los contratos se observan algunas inconsistencias, por ejemplo, en aspectos relacionados con los requisitos financieros, en donde las exigencias de indicadores como la rentabilidad sobre el patrimonio (ROE), la rentabilidad sobre activos (ROA), el índice de liquidez (IL) y el nivel de endeudamiento (NE) parecieran no estar relacionados con la experiencia del proponente o con la responsabilidad financiera del monto a ser contratado, es decir, al parecer estos indicadores se seleccionan de manera arbitraria al momento de establecer los términos de referencia de los contratos. Este hecho se evidencia en el cuadro 5 que muestra el comportamiento de estos indicadores en las empresas de Colombia.

Según esta información, por ejemplo para un proyecto de un valor aproximado a 62 salarios mínimos mensuales legales vigentes (smmlv) -como el contemplado en el proceso SECOP I (2019), que consiste en interventoría técnica y ambiental- los requerimientos de IL $\geq 30 \%, \mathrm{NE} \leq 5, \mathrm{ROE} \geq 22.50 \%$ y $\mathrm{ROA} \geq$ 19\% tan sólo son cumplidos de forma simultánea por el 6\% de las empresas colombianas, lo cual limita así la posibilidad de inclusión de otros posibles proponentes. Asimismo, para una interventoría con el mismo alcance para un contrato de un valor aproximado a $120 \mathrm{smmlv}$ (SECOP I, 2017), los requisitos habilitantes de contratación son cumplidos solamente por el $51 \%$ de las empresas colombianas. Es decir, se observa que no existe coherencia en las exigencias establecidas en función de los montos ni de las responsabilidades de las obras a ser supervisadas. 


\section{CUADRO 5}

Requisitos financieros de las empresas en Colombia

\begin{tabular}{|cccccccc|}
\hline ROE & $2015^{*} 1$ & ROA & $2015^{*} 1$ & IL & $2015^{* 1}$ & NE & $2015^{* 1}$ \\
\hline$<-0.20$ & $100 \%$ & $<-0.20$ & $100 \%$ & $<0.5$ & $100 \%$ & $10 \%$ & $6 \%$ \\
-0.2 & $95 \%$ & -0.2 & $98 \%$ & 0.5 & $97 \%$ & $20 \%$ & $14 \%$ \\
-0.1 & $90 \%$ & -0.15 & $95 \%$ & 1 & $91 \%$ & $30 \%$ & $24 \%$ \\
0 & $85 \%$ & -0.03 & $86 \%$ & 1.2 & $84 \%$ & $40 \%$ & $36 \%$ \\
0.05 & $73 \%$ & 0 & $84 \%$ & 1.5 & $75 \%$ & $50 \%$ & $51 \%$ \\
0.11 & $61 \%$ & 0.03 & $69 \%$ & 2 & $61 \%$ & $60 \%$ & $68 \%$ \\
0.19 & $46 \%$ & 0.06 & $59 \%$ & 3 & $44 \%$ & $70 \%$ & $82 \%$ \\
0.28 & $28 \%$ & 0.09 & $49 \%$ & 5 & $29 \%$ & $80 \%$ & $88 \%$ \\
0.3 & $24 \%$ & 0.12 & $38 \%$ & 10 & $15 \%$ & $90 \%$ & $94 \%$ \\
0.45 & $13 \%$ & 0.18 & $18 \%$ & 30 & $5 \%$ & $100 \%$ & $98 \%$ \\
0.5 & $9 \%$ & 0.2 & $11 \%$ & 80 & $2 \%$ & $>100 \%$ & $100 \%$ \\
\hline
\end{tabular}

Fuente: Cámara Colombiana de la Infraestructura (2016); Arias, Rengifo, Serrano Guzmán et al. (2018).

\section{Discusión}

Este artículo revela que durante la ejecución de los proyectos de construcción analizados se presentaron adiciones presupuestales y cambios en el cronograma de las obras debido a una aparente falta de control durante el ciclo de vida del proyecto. A pesar de que existía la figura del interventor, cuyo rol debería haber garantizado el cumplimiento contractual, pareciera ser que la falta de precisión en el alcance del ejercicio conlleva a situaciones anómalas durante la vigencia de los contratos que amparan la ejecución de las obras contratadas. En la tabla 1, adaptada de la propuesta por Chamorro Solórzano (2019), se proponen los alcances y controles recomendados para los diferentes tipos de interventoría, los cuales deberían ser incorporados en las normas y decretos que amparan la contratación estatal en Colombia:

\section{TABLA 1}

Alcance del interventor y las herramientas a considerar durante el ciclo de vida de los proyectos

\begin{tabular}{|c|c|c|c|c|c|c|c|c|c|c|}
\hline \multirow[b]{2}{*}{ Actividades del proyecto } & Inicio & \multicolumn{3}{|c|}{ Planeación } & \multicolumn{3}{|c|}{$\begin{array}{l}\text { Ejecución, moni- } \\
\text { toreo y control }\end{array}$} & \multicolumn{3}{|c|}{$\begin{array}{c}\text { Cierre o } \\
\text { liquidación }\end{array}$} \\
\hline & 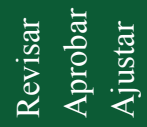 & 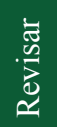 & $\begin{array}{l}\bar{\Xi} \\
0 \\
\text { 현 }\end{array}$ & 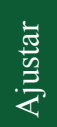 & $\begin{array}{l}\text { Z } \\
.0 \\
0 \\
0 \\
0\end{array}$ & 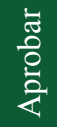 & 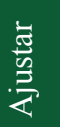 & $\frac{7}{3}$ & 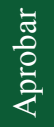 & 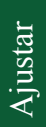 \\
\hline \multicolumn{11}{|l|}{ Interventoría técnica } \\
\hline Esquema básico, anteproyecto y diagnóstico & $\mathrm{X}$ & & & & & & & & & \\
\hline $\begin{array}{l}\text { Estudios y diseños, especificaciones técnicas, memorias de cálculo } \\
\text { de estudios y diseños, presupuestos, análisis de precios unitarios, } \\
\text { plan ambiental, plan social, plan y control de calidad, cronograma, } \\
\text { flujograma, certificación de equipos y/o maquinaria, almacenamiento } \\
\text { y certificación de materiales. }\end{array}$ & & $\mathrm{X}$ & $\mathrm{X}$ & & $\mathrm{X}$ & & $\mathrm{X}$ & & & \\
\hline Planos & & $\mathrm{X}$ & $\mathrm{X}$ & & $\mathrm{X}$ & & $\mathrm{X}$ & & & $\mathrm{X}$ \\
\hline Licencias o permisos & & $\mathrm{X}$ & & & & & & & & \\
\hline Localización de canteras de materiales o fuentes de suministros & & $\mathrm{X}$ & $\mathrm{X}$ & & $\mathrm{X}$ & & $\mathrm{X}$ & & & \\
\hline Seguridad y salud en el trabajo & & $\mathrm{X}$ & $\mathrm{X}$ & & & & $\mathrm{X}$ & & & \\
\hline Bitácora & & & & & $\mathrm{X}$ & $\mathrm{X}$ & $\mathrm{X}$ & & & \\
\hline Disponibilidad de servicios públicos & & $\mathrm{X}$ & & & & & & & & \\
\hline
\end{tabular}


TABLA 1 (continúa)

Alcance del interventor y las herramientas a considerar durante el ciclo de vida de los proyectos

\begin{tabular}{|c|c|c|c|c|c|c|c|}
\hline $\begin{array}{l}\text { Coordinación entre las partes involucradas (especialistas, asesores, } \\
\text { consultores) }\end{array}$ & $\mathrm{X}$ & $\mathrm{X}$ & $\mathrm{X}$ & $\mathrm{X}$ & $\mathrm{X}$ & & \\
\hline $\begin{array}{l}\text { Medición del impacto del proyecto, plan de mantenimiento y } \\
\text { sostenibilidad }\end{array}$ & & & $\mathrm{X}$ & $X$ & & $\mathrm{X}$ & $\mathrm{X}$ \\
\hline Verificación de hojas de vida y perfil del personal & $\mathrm{X}$ & $\mathrm{X}$ & $\mathrm{X}$ & $\mathrm{X}$ & & & \\
\hline Registro fotográfico & & & $\mathrm{X}$ & $\mathrm{X}$ & & & \\
\hline Identificación de riesgos (matriz) & $\mathrm{X}$ & $\mathrm{X}$ & $\mathrm{X}$ & & $\mathrm{X}$ & & \\
\hline \multicolumn{8}{|l|}{ Interventoría administrativa } \\
\hline Pólizas & & & $\mathrm{X}$ & $\mathrm{X}$ & $\mathrm{X}$ & $\mathrm{X} X$ & $\mathrm{X}$ \\
\hline Pago de seguridad social y parafiscales & & & $\mathrm{X}$ & $\mathrm{X}$ & & & \\
\hline $\begin{array}{l}\text { Actas parciales de obra, actas de ítems o actividades no previstas, } \\
\text { actas de comité técnico o reuniones, control de oficios o correspon- } \\
\text { dencia }\end{array}$ & & & $\mathrm{X}$ & $\mathrm{X}$ & & & \\
\hline Organigrama del proyecto, órganos e instancias de decisión & $\mathrm{X}$ & $\mathrm{X}$ & $\mathrm{X}$ & & $\mathrm{X}$ & & \\
\hline Contratación de fiducia o patrimonio autónomo & & & $\mathrm{X}$ & $\mathrm{X}$ & & & \\
\hline Informes de avance & & & $\mathrm{X}$ & $\mathrm{X}$ & $\mathrm{X}$ & & $\mathrm{X}$ \\
\hline $\begin{array}{l}\text { Trámites de prórrogas, adiciones al contrato, modificaciones al } \\
\text { contrato (otrosí) }\end{array}$ & & & $\mathrm{X}$ & $\mathrm{X}$ & $\mathrm{X}$ & & \\
\hline \multicolumn{8}{|l|}{ Interventoría financiera } \\
\hline Balance financiero del proyecto & & & $\mathrm{X}$ & $X$ & $\mathrm{X}$ & & \\
\hline $\begin{array}{l}\text { Saldos bancarios y/o fiduciarios, facturas de cobro parciales, intere- } \\
\text { ses o rendimiento financieros }\end{array}$ & & & $\mathrm{X}$ & $\mathrm{X}$ & & & \\
\hline \multicolumn{8}{|l|}{ Interventoría legal o jurídica } \\
\hline $\begin{array}{l}\text { Contratos principales, subcontratos, aplicación de multas y san- } \\
\text { ciones, verificación de obligaciones contractuales }\end{array}$ & & & X & $\mathrm{X}$ & & & \\
\hline \multicolumn{8}{|l|}{ Interventoría contable } \\
\hline $\begin{array}{l}\text { Contabilidad del proyecto, facturas, registros contables, extractos } \\
\text { contables y bancarios, comprobantes de egreso }\end{array}$ & & & $\mathrm{X}$ & $\mathrm{X}$ & & & \\
\hline \multicolumn{8}{|l|}{ Interventoría ambiental } \\
\hline Ejecución del plan de manejo ambiental, planes de compensación & & & $\mathrm{X}$ & $\mathrm{X}$ & $\mathrm{X}$ & & \\
\hline \multicolumn{8}{|l|}{ Interventoría social } \\
\hline Concertación con las comunidades & $\mathrm{X}$ & & $\mathrm{X}$ & $\mathrm{X}$ & & & \\
\hline $\begin{array}{l}\text { Comités de veeduría y control comunitario, plan de generación de } \\
\text { empleo }\end{array}$ & & & $\mathrm{X}$ & $\mathrm{X}$ & & & \\
\hline
\end{tabular}

Fuente: elaboración propia con información adaptada de Chamorro Solórzano (2019).

\section{Análisis Prospectivo}

El ejercicio de la ingeniería compromete el uso de diferentes recursos que se emplean para el cumplimiento de compromisos contractuales para la entrega de los productos, bienes o servicios de personas naturales, jurídicas, comunidades, etc. Este estudio reveló que la falta de definición en el rol del interventor en los procesos evaluados desencadenó en inconvenientes técnicos y financieros que terminan afectando al beneficiario del proyecto, bien o servicio contratado. Por ende, el estudio adelantado demuestra la necesidad de replantear la normatividad que ampara el ejercicio de la actividad de ingeniería en Colombia desde el punto de vista de la interventoría, en donde el rol de acción del ingeniero se deslinda en ocasiones de las reales obligaciones para el buen logro de los proyectos que se acometen en las condiciones de presupuesto y tiempo pactados. Se plantea la importancia del papel del interventor a lo largo del ciclo de vida del proyecto que se contratará. Los resultados obtenidos constituyen un llamado de atención al gobierno central y, en lo particular, a la rama legislativa para que se revisen los alcances de la labor de interventoría incluyendo los perfiles idóneos para cada una de las actividades. 


\section{Conclusiones}

La ejecución de un proyecto de construcción exige previamente una adecuada planeación de actividades, lo que conlleva a la determinación del precio y del tiempo que deben estar explícitos en el contrato correspondiente. Además, es claro que todo proyecto debe cumplir un ciclo de vida que debe abarcar desde el inicio hasta su cierre o liquidación. Cuando se contratan los servicios de un interventor, dependiendo del alcance de la labor que se va a contratar, existen responsabilidades o acciones de control y supervisión que, como mínimo, deberían hacerse cumplir.

En el caso particular de este estudio, adelantado en el Valle del Cauca (Colombia), se encontró que nada más el $15.63 \%$ de los 64 contratos de interventoría fue ejecutado en los tiempos y al presupuesto pactado. Asimismo, se evidenció que el alcance del rol del interventor en los procesos estudiados no es claro o presentó ambigüedades a tal punto que el $80 \%$ incluía funciones relacionadas con aspectos de tipo técnico, administrativo y el $50 \%$ de los contratos contemplaba aspectos jurídicos o legales, y en un solo proceso interventoría ambiental y social. Por otro lado, en tres de los procesos ocurrieron adiciones presupuestales sin afectaciones al cronograma de entrega, en 18 se encontraron adiciones presupuestales y prórrogas para la finalización y uno fue ejecutado en menor tiempo y presupuesto pactado.

Dentro de los hallazgos relevantes de estos procesos pueden mencionarse: cambio del alcance en la ejecución del proyecto inicialmente contratado, ausencia o baja calidad de los estudios y diseños del proyecto con el consecuente incremento en costos y variaciones en el cronograma, uso inapropiado de fondos de anticipo, adiciones presupuestales y de tiempo, principalmente. En otro aspecto, se observó que los requisitos financieros definidos por el ente contratante para proyectos similares no son consistentes con el monto ni con el objeto contractual por lo cual pudieran presentarse procesos donde se posibilita la participación a un número reducido de proponentes.

Este artículo enfatiza la importancia del rol del interventor de Colombia en la etapa de planeación de un proyecto de infraestructura pública en donde se debe concretar el alcance de la labor que se va a ejecutar, definición del cronograma, preparación del presupuesto e identificación de las reservas presupuestales para la ejecución, especificaciones de calidad, planteamiento de estrategias de comunicación, entre otros aspectos.

Resulta evidente que la legislación colombiana relacionada con la contratación pública debe ser revisada y ajustada de modo que sean explícitos los alcances y perfiles adecuados para el ejercicio de la labor de interventoría. En los procesos analizados, como se mencionó, se encontró ausencia de cumplimiento de esta normatividad por cuanto son significativos los hallazgos relevantes sin la correspondiente sanción o multa a los infractores por estas modificaciones, reclamaciones del contratista de obra por desequilibrio contractual, desembolso de anticipo sobre un concepto no permitido, entre otros más. Este último hecho, por ejemplo, constituye en una incidencia fiscal, disciplinaria y penal, lo que corrobora la necesidad de mejorar los procesos actuales de interventoría que se ejercen como exigencia al seguimiento y control de los proyectos.

\section{Agradecimientos}

Los autores agradecen los aportes realizados por los evaluadores, ya que permitieron los ajustes técnicos necesarios para lograr la publicación, pero se deslindan las responsabilidades habituales.

\section{REFERENCIAS}

Agencia Nacional de Infraestructura. (2013). Guía de buenas prácticas para interventoria. Bogotá D. C.

Alcaldía de Medellín. (3 de octubre de 2014). Portal del Ciudadano. Disponible en https://www.medellin. gov.co/irj/go/km/docs/pccdesign/SubportaldelCiudadano_2/PlandeDesarrollo_0_15/Noticias/Shared\%20Content/Documentos/2014/Uniandes_Informe-Final-Fase3-SPACE-Resumen.pdf 
Arias, C., Rengifo, D., Serrano Guzmán, M., Pérez Ruiz, D., \& Torrado Gómez, L. (2018). Análisis de los requisitos habilitantes de contratación pública en proyectos de infraestructura vial terminados anormalmente después de convocados. Revista de Obras Públicas, 3598.

Cámara Colombiana de la Infraestructura. (2016). Documento de buenas prácticas: licitación pública. Colombia. Disponible en http://www.infraestructura.org.co/descargas/Licitacion.pdf

Chamorro Solórzano, G. (2019). Evaluación de la gestión del interventor en proyectos de infraestructura pública en el Departamento del Valle del Cauca desde la expedición del estatuto de Anticorrupción (trabajo de grado de maestría dirigido por la ingeniera María Fernanda Serrano G.). Pontificia Universidad Javeriana Cali. Santiago de Cali.

Congreso de Colombia. (1993). Ley 80 del 28 de octubre 1993, por la cual se expide el Estatuto General de Contratación de la Administración Pública. Bogotá D. C.

Congreso de Colombia. (2011). Ley 1474 del 12 de julio de 2011, por la cual se dictan normas orientadas a fortalecer los mecanismos de prevención, investigación y sanción de actos de corrupción y la efectividad del control de la gestión pública. Bogotá D. C.

Congreso de Colombia. (2018). Ley 1882 del 15 de enero de 2018. Santa Fe de Bogotá: Congreso de la República de Colombia.

Contraloría General de La República. (2014). Informe Actuación Especial No. 018-2014 (1 de julio de 2014). Disponible en http://www.contraloriagen.gov.co/documents/10136/186575321/INFORME_FINAL_ACES_DEPARTAMENTO+VALLE+DEL+CAUCA+RECURSOS+FNR+Y+SGR+VIGENCIAS+2011-2013.PDF/1c7558e7-36c3-4d95-8520-7afc3192237a?version=1.0

Empresas Municipales de Cali. (2014). Supervisión y control de la contratación de EMCALI. Santiago de Cali.

Gorbaneff, Y., González, J. M. y Barón, L. (2011). ¿Para qué sirve la interventoría de las obras públicas en Colombia? Revista de Economia Institucional, 13(24), 413-428.

Instituto de Desarrollo Urbano. (2015). Disponible en https://www.idu.gov.co/documents/20181/1990925/ MGEO01_INTERVENTORIA_Y_O_SUPERVISION_DE_CONTRATOS_V_3.0.pdf/9a4eb48e-1912-413c-b302-04f3dfd8be66

Instituto Nacional de Vías. (2010). Manual de interventoria obra pública. Bogotá D. C.

National Research Council Canada. (2009). Audit of construction contracts. Retrieved from https://www. nrc-cnrc.gc.ca/obj/doc/about-apropos/planning_reporting-planification_rapports/audit-verification/ construction-eng.pdf

Ottawa Documents. (2012). Audit of contruction supervision 2012. Vérification de la surveillance de la construction. Retrieved from http://documents.ottawa.ca/sites/documents.ottawa.ca/files/documents/audit_2012_ construction.pdf

Semana. (2010). La caida del Grupo Nule. Disponible en http://www.semana.com/nacion/articulo/la-caidadel-grupo-nule/121790-3

Sánchez Henao, J. (2007). Gestión organizativa en el proceso edificatorio: regulación de la interventoria de proyectos en Colombia (tesis de doctorado). Madrid: Universidad Politécnica de Madrid.

SECOP I. (2019). CM CA 002 2019. Subachoque: Servicio Electrónico de Contratación Pública. Disponible en https://www.contratos.gov.co/consultas/detalleProceso.do?numConstancia=19-15-9381302

SECOP I. (2017). CMA-DO-SRT-082-2017. La Guajira: Servicio Electrónico de Contratación Pública. Disponible en https://www.contratos.gov.co/consultas/detalleProceso.do?numConstancia=17-15-6989060

SECOP I. (2015a).330-15-08-027. Tuluá: Servicio Electrónico de Contratación Pública. Disponible en https:// 
www.contratos.gov.co/consultas/detalleProceso.do?numConstancia=15-15-4309027

SECOP I. (2015b). FEV-CM-003-2015. Santiago de Cali: Servicio Electrónico de Contratación Pública. Disponible en https://www.contratos.gov.co/consultas/detalleProceso.do?numConstancia=15-15-3978977

SECOP I. (2015c). 2015-154. El Dovio: Servicio Electrónico de Contratación Pública. Disponible en https:// www.contratos.gov.co/consultas/detalleProceso.do?numConstancia=15-15-4242353

SECOP I. (2015d). PSCMA-083-2015. Cartago: Servicio Electrónico de Contratación Pública. Disponible en https://www.contratos.gov.co/consultas/detalleProceso.do?numConstancia=15-15-4251876

SECOP I. (2015e). 330-15-08-013. Tuluá: Servicio Electrónico de Contratación Pública. Disponible en https:// www.contratos.gov.co/consultas/detalleProceso.do?numConstancia=15-15-3964384

SECOP I. (2015f). 330-15-08-014. Tuluá: Servicio Electrónico de Contratación Pública. Disponible en https:// www.contratos.gov.co/consultas/detalleProceso.do?numConstancia=15-15-3973821

SECOP I. (2015g). PSCMA-072-2015. Cartago: Servicio Electrónico de Contratación Pública. Disponible en https://www.contratos.gov.co/consultas/detalleProceso.do?numConstancia=15-15-4196471

SECOP I. (2015h). PSCMA-071-2015. Cartago: Servicio Electrónico de Contratación Pública. Disponible en https://www.contratos.gov.co/consultas/detalleProceso.do?numConstancia=15-15-4192896

SECOP I. (2015i). 4162.0.14.10.002-2015. Santiago de Cali: Servicio Electrónico de Contratación Pública. Disponible en https://www.contratos.gov.co/consultas/detalleProceso.do?numConstancia=15-15-3677588

SECOP I. (2015j). CMA-IMVY-001-2015. Yumbo: Servicio Electrónico de Contratación Pública. Disponible en https://www.contratos.gov.co/consultas/detalleProceso.do?numConstancia=15-15-4093367

SECOP I. (2015k). MC-915.108.5.04.15. Santiago de Cali: Servicio Electrónico de Contratación Pública. Disponible en https://www.contratos.gov.co/consultas/detalleProceso.do?numConstancia=15-15-3865192

SECOP I. (2014a). 4133.0.32.076-2014. Santiago de Cali: Servicio Electrónico de Contratación Pública. Disponible en https://www.contratos.gov.co/consultas/detalleProceso.do?numConstancia=14-15-2955474

SECOP I. (2014b). CM-SI-027-2013. Tuluá: Servicio Electrónico de Contratación Pública. Disponible en https://www.contratos.gov.co/consultas/detalleProceso.do?numConstancia=14-15-2899971

SECOP I. (2014c). 330-015-008-020. Tuluá: Servicio Electrónico de Contratación Pública. Disponible en https://www.contratos.gov.co/consultas/detalleProceso.do?numConstancia=14-15-2745087

SECOP I. (2014d). CM-SI-004-2014. Yumbo: Servicio Electrónico de Contratación Pública. Disponible en https://www.contratos.gov.co/consultas/detalleProceso.do?numConstancia=14-15-3036418

SECOP I. (2014e). 4133.0.32.020-2014. Santiago de Cali: Servicio Electrónico de Contratación Pública. Disponible en https://www.contratos.gov.co/consultas/detalleProceso.do?numConstancia=14-15-2807 107

SECOP I. (2014f). FEV-CM-004-2014. Santiago de Cali: Servicio Electrónico de Contratación Pública. Disponible en https://www.contratos.gov.co/consultas/detalleProceso.do?numConstancia=14-15-3037436

SECOP I . (2014g). 330-015-008-004. Tuluá: Servicio Electrónico de Contratación Pública. Disponible en 330-015-008-004.

SECOP I. (2014h). 008-SG-2014. Palmira: Servicio Electrónico de Contratación Pública. Disponible en https:// www.contratos.gov.co/consultas/detalleProceso.do?numConstancia=14-15-3051988

SECOP I . (2014i). MP-SDRU-CM-16-2014. Palmira: Servicio Electrónico de Contratación Pública. Disponible en https://www.contratos.gov.co/consultas/detalleProceso.do ?numConstancia=14-15-3094103

SECOP I. (2013a). 330-015-008-022. Tuluá: Servicio Electrónico de Contratación Pública. Disponible en https://www.contratos.gov.co/consultas/detalleProceso.do?numConstancia=13-15-1690511 
SECOP I. (2013b). MV-CM-002 DE 2013. Vijes: Servicio Electrónico de Contratación Pública. Disponible en https://www.contratos.gov.co/consultas/detalleProceso.do?numConstancia=13-15-1898742

SECOP I. (2013c). CM-FEV-001-2013. Santiago de Cali: Servicio Electrónico de Contratación Pública. Disponible en https://www.contratos.gov.co/consultas/detalleProceso.do?numConstancia=13-15-1973804

SECOP I. (2013d). 001-2013- OOPP -CONCURSO DE MERITOS. Ríofrío: Servicio Electrónico de Contratación Pública. Disponible en https://www.contratos.gov.co/consultas/detalleProceso.do?numConstancia $=13-15-2095624$

SECOP I. (2013e).2013-187. Caicedonia: Servicio Electrónico de Contratación Pública. Disponible en https:// www.contratos.gov.co/consultas/detalleProceso.do?numConstancia $=13-15-2095770$

SECOP I. (2013f). CM-SI-012-2013. Yumbo: Servicio Electrónico de Contratación Pública. Disponible en https://www.contratos.gov.co/consultas/detalleProceso.do?numConstancia=13-15-1965202

SECOP I. (2013g). 330-015-008-060. Tuluá: Servicio Electrónico de Contratación Pública. Disponible en https://www.contratos.gov.co/consultas/detalleProceso.do?numConstancia=13-15-2109797

SECOP I. (2013h). 330-015-008-006. Tuluá: Servicio Electrónico de Contratación Pública. Disponible en https://www.contratos.gov.co/consultas/detalleProceso.do?numConstancia=13-15-1602577

SECOP I. (2013i). CM-SI-031-2013. Yumbo: Servico Electrónico de Contratación. Disponible en https:// www.contratos.gov.co/consultas/detalleProceso.do?numConstancia=13-15-2116058

SECOP I. (2013j). CM 11 DE 2013. Cali: Servicio Electrónico de Contratación Púbilica. Disponible en https:// www.contratos.gov.co/consultas/detalleProceso.do?numConstancia=13-15-1797857

SECOP I. (2013k). PCMCST-001-2013. Toro: Servicio Electrónico de Contratación Pública. Disponible en https://www.contratos.gov.co/consultas/detalleProceso.do?numConstancia=13-15-1988423

SECOP I. (20131). 330-015-008-039. Tuluá: Servicio Electrónico de Contratación Pública. Disponible en https://www.contratos.gov.co/consultas/detalleProceso.do?numConstancia=13-15-1914698

SECOP I. (2012a). CM 01 DE 2012. Santiago de Cali: Servicio Electrónico de Contratación Pública. Disponible en https://www.contratos.gov.co/consultas/detalleProceso.do?numConstancia=12-15-1239626

SECOP I. (2011a). MC-CM-007-2011. Buenaventura: Servicio Electrónico de Contratación Pública. Disponible en https://www.contratos.gov.co/consultas/detalleProceso.do?numConstancia=11-15-512440

SECOP I. (2011b). MC-5.8.7.03.11. Santiago de Cali: Servicio Electrónico de Contratación Pública. Disponible en https://www.contratos.gov.co/consultas/detalleProceso.do?numConstancia=16-15-4818407

SECOP I. (2011c). MC-CM-026-2011. Santiago de Cali: Servicio Electrónico de Contratación Pública. Disponible en https://www.contratos.gov.co/consultas/detalleProceso.do?numConstancia=11-15-685009

SECOP I. (2011d). CM 09 DE 2011. Vijes, Santiago de Cali: Servicio Electrónico de Contratación Pública. Disponible en https://www.contratos.gov.co/consultas/detalleProceso.do?numConstancia=11-15-696840

SECOP I. (2010a). MC-CM-001-2010. Buenaventura: Servicio Electrónico de Contratación Pública. Disponible en https://www.contratos.gov.co/consultas/detalleProceso.do?numConstancia=10-15-303830

Serrano Guzmán, M., Carmona Escobar, J., Pérez Ruíz, D. y Potes Patiño, J. (2019). Causales para la terminación anormal del proceso después de convocada la licitación pública en el Valle del Cauca (2013-2016). Revista Via Iuris, 26, 1-30. Disponible en https://revistas.libertadores.edu.co/index.php/ViaIuris/article/view/867/845

Servicio Electrónico de Contratación Pública. (2016). Portal de Colombia Compra Eficiente. Disponible en: https://www.colombiacompra.gov.co/proveedores/consulte-en-el-secop-i.

Universidad Industrial de Santander. (2014). Disponible en https://www.uis.edu.co/intranet/calidad/documentos/contratacion/Manuales/MCO.01.pdf 


\section{Notas}

[1] El art. 5 de la Ley 1474 de 2011 establece las inhabilidades para contratar en entidades públicas y el art. 44 señala los sujetos disciplinables. El art. 45, sobre la responsabilidad del interventor por faltas gravísimas, fue derogado por el art. 265 de la Ley 1952 de 2019.

[2] Según Gorbaneff, González y Barón (2011: 413), “aunque la interventoría fue diseñada para blindar el Estado es una de las fisuras por las que entra la corrupción".

[3] En oficio del 31 enero de 2014 el Cuerpo de Bomberos de la ciudad de Santiago de Cali hace señalamientos a la red contraincendio, a los "trabajos inherentes al presente contrato [...] se requiere suspender el contrato [...] hasta que se den las condiciones de seguridad para las personas, la protección del bien y la continuidad de las operaciones” (Chamorro Solórzano, 2019).

[4] Según el hallazgo Núm. 4 Manejo de Anticipo descrito en el Informe de Actuación Especial de Fiscalización por parte de la Contraloría General de Santiago de Cali, con lo cual se transgreden presuntamente los artículos 83 y 84 de la Ley 1474 de 2011 y el artículo 34 de la Ley 734 de 2002 que ocasiona un presunto detrimento patrimonial. Presunta incidencia fiscal, disciplinaria y penal.

[5] En el acta de liquidación del proceso, el contratista deja una anotación referente al desequilibrio económico por "no incumplir la cantera

\section{BY-NC-ND}

